Zeitschrift für Augenheilkunde 1923;50:I-IV

\title{
Contents, Vol. 50, 1923
}

Inhalts - Verzeichnis.

Eigen-Arbeiten. seite

Aust, 0., v. Hippelsche Erkrankung mit Stauungspapille

und Hämangiom 305

Bergmeister, R., Temporäre Einwärtskehrung der Ziliender Unterlider, bedingt durch

Muskelspasmus, ohneEntropium 153

Birch-Hirsch†eld, A., Nochmals zur Schädigung des Auges

durch Röntgenstrahlen 135

- - Zur pathologischen Anatomie des Trachoms, be-

sonders in den Spätstadien 261

Clausen, W. und J. Bauer, Beiträge und Gedanken zur

Lehre von der Vererbung des Strabismus concomitans 313

Gilbert, W., Über Uveitis 267

Guist, G., Ernährungsverhältnisse der Netzhaut und Ader-haut des Rattenauges. Bericht über 211

von Kopp > any itransplantierte Rattenaugen195

Hartig, F., Über den gegenwärtigen Stand der Behand-

lung der Augentuberkulose 79

Jacoby, J., Die pathologisch-anatomischen Grundlagender Pseudogliome nebst Bemerkungen zur Differential-diagnose von Gliom und Pseudogliom 95

Klainguti, R,, Anatomischer Befund bei Wiederanlegungeiner posttraumatisch abgelösten

Netzhaut. (HierzuTafel IV) 71

Knüsel, 0., Vitale Färbungen am menschlichen Auge II.

(Hierzu Tafel I/III) 23

Kramer, R., Zur Analogie der Prismen- und Zylinderwir-

kung 181

- $\quad$ Technische Verbesserungen zur Zylinderskiaskopie 297

Kraupa, E., Die Retinitis centralis anularis 335

Kraupa-Runk, M., Prinzipien zur Klinik und Therapie des

Herpes corneae 345

Meller, J., Über die Perineuritis und Periarteritis ciliaris

bei Herpes zoster ophthalmicus 2

Mennerich, P., Ein Fall von Retraktionsbewegungen derAugen bei angeborener Anomalie der äußeren Augen-muskeln 173

$-\mathrm{IV}-$

Seite

Oswald, Beiträge zur Klinik des Ringskotoms .... 39

Urbanek, J., Ein klinischer Beitrag zur Frage der Vossius-

schen Ringtrübung 159

Vogt, A., Neue Beobachtungen über menschliche Kristall- 
linsen mit doppeltem Brennpunkt 145

Wachtler, G., Über van der Hoeves Verfahren zur Verhütungvon Glaskörpervorfall nebst

Bemerkungen über sub-konjunktivale Anästhesie 359

Merz Weigandt, Frühzeitiges Auftreten von Metastaseund Ringabszeß der Hornhaut bei

kryptogenetischerSepsis $\quad 357$

- $\quad$ - Weiterer Beitrag zu den pflanzlichen Fremdkörpern

in der Bindehaut 355

Mitteilungen aus der Praxis fur die Praxis.

Kuhnt, H., Extraktion eines Geschosses aus der mittleren

retrobulbären Orbita 103

- $\quad$ - Chronischer Schwund der Lidfixationen .... $\quad$ 106Schott, -ST., Keratitis

parenchymatosa und Salvarsan . . 109Avizonis, P., Aufhellung einer Kontusionskatarakt ... 113

Bericht über die deutsche ophthalmologische Literatur.

Untersuchungsmethoden (I. u. II. Semester 1922). Von

Dr. B. Kramer in Wien

367

Gesellschaftsberichte.

Ophthalmologische Gesèllschaft in Wien. Sitzung vom

11. XII 1922 115

Sitzung vom 15. I. 1923

Sitzung vom 22. I. 1923

Sitzung vom 19. II. 1923

Ungarische Ophthalmologische Gesèllschaft. Sitzungs-

berichte vom 5. November und 3. Dezember 1922 . 248

Berliner Augenärztliche Gesèllschaft vom 22. II. 1923 . . 251

Diagnose und Therapie 122, 253, 375

Aus physiologischen Zeitschriften 380

Buchbesprechungen 133

P. Personalien 134

Tagesnachrichten 133 